EPJ Web of Conferences 43, 01004 (2013)

DOI: $10.1051 /$ epjconf/20134301004

(C) Owned by the authors, published by EDP Sciences, 2013

\title{
Why GN93 should not be used anymore
}

\author{
N. Grevesse ${ }^{1, a}$, M. Asplund ${ }^{2}$, J. Sauval ${ }^{3}$ and P. Scott ${ }^{4}$ \\ ${ }^{1}$ Centre Spatial de Liège and Institut d'Astrophysique et de Géophysique, Université de \\ Liège, Belgium \\ ${ }^{2}$ Australian National University, Research School of Astronomy and Astrophysics, Weston \\ Creek, Australia \\ ${ }^{3}$ Observatoire Royal de Belgique, Bruxelles, Belgium \\ ${ }^{4}$ Department of Physics, McGill University, Montréal, Canada
}

\begin{abstract}
We show why the solar chemical composition of [1] (GN93) and the similar values of [2] (AG89) and [3] (GS98), characterized by values of the metallicity of the order of 0.017 to 0.020 , largely used in solar and stellar modeling, are now obsolete. They should be replaced by the new and more precise results of [4] (AGSS09), with a much lower metallicity of 0.0134 .
\end{abstract}

\section{INTRODUCTION}

We know that the solar metallicity, Z, is essentially dominated by oxygen (43\%), carbon (18\%), neon $(10 \%)$ and iron $(10 \%)$. As the solar abundance of $\mathrm{Ne}$, obtained from the $\mathrm{Ne} / \mathrm{O}$ ratio in the corona, depends directly on the abundance of $\mathrm{O}$, we shall essentially discuss the evolution of the abundances of oxygen and carbon, the two main contributors to $\mathrm{Z}$.

\section{THE “OLD” SOLAR MIXTURES (AG83, GN93, GS98)}

In the solar chemical compositions recommended by [2] (AG89), [1] (GN93) and [3] (GS98), the abundances of $\mathrm{O}$ and $\mathrm{C}$ were determined from essentially the same indicators as the ones used by [4] (AGSS09) i.e. OI and CI permitted and forbidden lines as well as molecular lines of $\mathrm{OH}, \mathrm{CH}$ and C2. The solar photospheric model was the 1D model of [5] largely used in those days. The solar data were essentially the same as the ones used nowadays. The main characteristics of the results of the three above mentioned solar mixtures and the evolution from AG89 $(\mathrm{Z}=0.02)$ to GN93 $(\mathrm{Z}=0.018)$ to GS98 $(Z=0.017)$ are the following: the results from the various indicators, permitted and forbidden atomic lines and molecular lines, agree within about 0.1 dex and the evolution from 1989 to 1998 is essentially due to progress in the atomic and molecular data used to derive the abundances.

\section{THE AGSS09 SOLAR MIXTURE}

AGSS09 revised the chemical composition of all the elements in the sun. This is the first comprehensive and homogeneous work on solar abundances since many decades. The same rules were applied for all the elements. We used a new 3D model of the solar outer layers instead of the old 1D model mentioned

\footnotetext{
ae-mail: Nicolas.Grevesse@ulg.ac.be
}

This is an Open Access article distributed under the terms of the Creative Commons Attribution License 2.0, which permits unrestricted use, distribution, and reproduction in any medium, provided the original work is properly cited. 
above. This new 3D model really outperforms the 1D model: it passes successfully a series of tests where the 1D model fails. For example, for the first time, we were able to reproduce the observed asymmetries in the profiles of the spectral lines. We also made a very careful and very demanding selection of the spectral lines. We definitely preferred quality over quantity. Using lines that are blended, even slightly, increases the dispersion of the results and skews the mean abundance results to higher values. We also replaced the hypothesis of local thermodynamic equilibrium (LTE) used in older analyses by non-LTE analyses when possible i.e. when the required large number of atomic data was available. In addition, for $\mathrm{C}, \mathrm{N}$ and $\mathrm{O}$ we used all the indicators, atoms as well as molecules, as in the old analyses.

\section{INDICATORS OF THE ABUNDANCE OF OXYGEN}

In order to illustrate the recent decrease in the solar $\mathrm{Z}$, we shall examine the results of the $\mathrm{O}$ abundance obtained by GN93 and compare them with AGSS09. The available indicators of the abundance of O are three forbidden lines of OI with very low excitation energies, a few very high excitation (larger than $9 \mathrm{eV}$ ) permitted $\mathrm{OI}$ lines and a very large number of vibration-rotation and pure rotation lines of $\mathrm{OH}$, very clean and easy to measure with accuracy. It is easy to show that, at solar photospheric temperatures, most of the $\mathrm{O}$ is in the lower OI levels. That means that the forbidden lines are not sensitive to departures from LTE and not very sensitive to the temperature i.e. to the model. The very high excitation permitted OI lines with level population of about $10^{-9}$ are on the contrary extremely sensitive to departures from LTE compared with the ground levels. It can also be easily shown that the molecular lines are not more sensitive to the temperature than the high excitation OI lines. The only difference is that they are formed in somewhat higher layers than the atomic lines.

\section{DIFFERENCES BETWEEN GN93 AND AGSS09}

The abundance derived from the three forbidden lines by GN93 is high (8.95). In the meantime it has been discovered that each of these ideal indicators of the $\mathrm{O}$ abundance is unfortunately blended. AGSS09 took the blends very carefully into account, in a purely empirical way, independent of any atmospheric model. This leads to a decrease of the $\mathrm{O}$ abundance to 8.69. The impact of the use of the 3D model rather than the $1 \mathrm{D}$ model atmosphere is negligible.

The handful of high excitation OI lines were treated in LTE by GN93. We already suspected possible departures from LTE, but the required atomic data to compute the lines in non-LTE were not available. The result of GN93 is an abundance of $\mathrm{O}$ of 8.83, somewhat lower than the value obtained from the forbidden lines. AGSS09 were able to treat the non-LTE excitation of OI lines with great accuracy. They were able to calibrate one of the poorly known data in the non-LTE calculations, namely the crosssections for the collisions with the very abundant neutral hydrogen atoms in the photospheric layers. They calibrated these cross-sections from very precise center to limb observations of a few of these high excitation O I lines. The very large non-LTE corrections to the LTE abundances are negative and the AGSS09 O abundance value decreased to 8.69. The impact of the new 3D model is also very small.

The only indicators that are very sensitive to the use of a $3 \mathrm{D}$ rather than a $1 \mathrm{D}$ model atmosphere are the molecules. The mean temperature of the 3D model in the outer layers, where the molecular lines are formed, appears to be somewhat smaller than the corresponding temperature in the 1D model. Therefore, the 3D AGSS09 result from the OH lines is smaller (8.69) than the 1D value of GN93 (8.87). We also showed that if the empirical 1D model of [5] used by GN93 were reconstructed today with spectral lines measured on spectral atlases with larger resolving power than the one originally used, the temperature in the outer solar layers would be lower, in agreement with the present mean temperature of the 3D model. The lowering of the abundance derived from the molecular lines is clearly a consequence of the use of the 3D model.

Let us summarize the differences between new and old results: the decrease of the abundances from the forbidden lines is due to the presence of blends that were unknown 15 years ago, the decrease of 


\section{Ageing Low Mass Stars: From Red Giants to White Dwarfs}

the abundance from the high excitation permitted OI lines is due to the departures from LTE affecting those lines, effects that could not be computed 15 years ago because of the lack of the required atomic data, and finally the decrease of the abundance from the molecular lines is really the result of the new 3D model. The new final abundances from the 3 different indicators are now in perfect agreement. The old values also agreed but with much larger differences between the various indicators.

\section{ANOTHER RECENT SOLAR MIXTURE BY CAFFAU ET AL. [6]}

Caffau et al. ([6] and references therein) have also revisited the solar abundances of a few elements using their own 3D model of the solar photosphere. The results from these authors are slightly larger than AGSS09, e.g. O [6]= 8.76 compared with 8.69 (AGSS09) and C [6] = 8.50 compared with 8.43 (AGSS09). We have been able to identify the main reasons for these differences: they are not at all related to the use of different $3 \mathrm{D}$ models. Indeed these models agree and the differences in abundances are always lower than about 5\%. If we examine the $\mathrm{O}$ results, only based on the atomic lines in [6], we see that their somewhat larger value from the forbidden lines should decrease if the blends were more accurately estimated. The value from the permitted lines could also somewhat decrease if their solar data as well as the non-LTE results were updated. For carbon, the dispersion in their results is clearly produced by the use of many strong CI lines that are slightly blended leading to a somewhat too large abundance.

\section{CONCLUSIONS AND REMARKS}

One of us (NG) thanks the many of you who used our past solar mixtures, AG89, GN93 and GS98. However we now advise to forget about these old day solar mixtures and now use our most recent results AGSS09.

Very recently, new 3D models have been built that take solar photospheric magnetic fields into account ([7] and [8]). These new models might have an influence on the solar abundances. First estimates of their impact on the $\mathrm{O}$ abundance for example, are a very small increase for the results from the atomic lines and a somewhat larger increase on the abundances derived from the molecular lines. We however need to wait for more severe tests of these new 3D models before being able to really quantify their actual impacts on solar abundances.

NG thanks Josefina Montalbán and Arlette Noels for their help and for insisting to clarify the differences between "Now" and the "Past".

\section{References}

[1] N. Grevesse, A. Noels, Origin and evolution of the elements (Eds. N. Prantzos, E. Vangioni-Flam, M. Cassé, Cambridge University Press) 15 (1993)

[2] E. Anders, N. Grevesse, Geochimica et Cosmochimica Acta 53, 197 (1989)

[3] N. Grevesse, J. Sauval, Space Sci. Rev. 85, 161 (1998)

[4] M. Asplund, N. Grevesse, J. Sauval, P. Scott, Ann. Rev. Astron. Astrophys. 47, 481 (2009)

[5] H. Holweger, E.A. Müller, Solar Phys. 39, 19 (1974)

[6] E. Caffau, H.-G. Ludwig, M. Steffen, B. Freytag, P. Bonifacio, Solar Phys. 268, 255 (2011)

[7] D. Fabbian, E. Khomenko, F. Moreno-Insertis, A. Nordlund, Astrophys. J. 724, 1536 (2010)

[8] D. Fabbian, F. Moreno-Insertis, E. Khomenko, A. Nordlund, Astron. Astrophys. arXiv:1209.2771 (2012) 